\title{
Ethical implications of array comparative genomic hybridization in complex phenotypes: points to consider in research
}

\author{
Holly K. Tabor, PhD, and Mildred K. Cho, PhD
}

\begin{abstract}
As with many new diagnostic technologies, the recent rapid emergence of array comparative genome hybridization in clinical genetics provides the power to observe new biological phenomena before their clinical significance is well understood. This raises ethical issues for clinicians when applying the technologies. However, at this early stage of research and development on array comparative genome hybridization, the ethical implications of the conduct of research, as well as how research findings are presented and interpreted, should also be considered by the research, clinical, and ethics communities. These considerations are especially important in the use of array comparative genome hybridization to study complex and common traits. We examined recent publications on autism as an example of the application of array comparative genome hybridization to a complex phenotype. Our goal was to identify points to consider for researchers, clinicians, and patients/families to ensure responsible and ethical design, presentation, and interpretation of these kinds of studies. Genet Med 2007:9(9):626-631.
\end{abstract}

Key Words: aCGH, prenatal genetic testing, ethics, complex traits, autism

\section{INTRODUCTION}

Microarrays have now entered the realm of clinical diagnostics for a number of applications, including prenatal genetic testing. In this article, we focus on ethical issues in the application of microarrays to the study of complex traits, an area of increasing research that is only in the earliest stages of potential clinical application. Because complex traits are often more prevalent in the population than Mendelian traits, the use of microarrays for diagnostic purposes has much broader ethical and societal implications.

As with the advent of many new diagnostic technologies in medicine, the use of microarrays has provided unprecedented power to detect biochemical, morphological, and physiological differences, sometimes of unknown or unconfirmed clinical significance. This power also creates challenges that have important ethical implications that should be considered. Many of the ethical issues raised by microarrays are not new. For example, clinical geneticists and genetic counselors have extensive experience thinking and communicating about ethical issues with their patients, and many of the issues in microarrays parallel issues involved with other genetic diagnostic

From the Stanford Center for Biomedical Ethics, Department of Pediatrics, Stanford University School of Medicine, Palo Alto, California.

Holly K. Tabor, 701 Welch Road, Suite A1105, Palo Alto, CA 94304-5748; E-mail: hktabor@stanford.edu

Disclosure: The authors report no conflict of interest.

Submitted for publication May 7, 2007.

Accepted for publication June 20, 2007.

DOI: 10.1097/GIM.0b013e3181485688 techniques. Furthermore, many of the ethical issues involved with the application of microarray technologies exist broadly for many new diagnostic technologies.

The use of microarrays raises at least four issues that exist broadly for many new diagnostic technologies. Although the ethical issues in microarray application to complex traits may not all be novel, we suggest that these issues merit consideration and reexamination in this context. First, do we understand normal variation enough to know what is "abnormal" or "pathogenic"? If not, use of the tests could cause more harm than good to patients and their families because of the probability that they provide inaccurate information that may lead to unnecessary anxiety, clinical procedures, or even termination of pregnancy. ${ }^{1}$ Second, because new technologies like microarrays allow inferences to be made about traits that are not necessarily associated with morbidity or mortality, or traits that are nonmedical (such as personality or hair color), there is concern that the availability of the technology may increase pressure on prospective parents and clinicians to screen for "imperfections" to eliminate them. ${ }^{2}$ Such screening raises the specter of eugenics ${ }^{3}$ and raises questions about the appropriate role of the clinician in providing information that is not about health and disease.

Third, new powerful technologies, like the comprehensive and detailed analysis of the genome afforded by microarray analysis, creates a greater potential for incidental findings. Incidental findings are those that are identified by the application of a new technology that were not part of the original scope of what was being examined. This is a common theme of new diagnostic technologies: their ever-increasing ability to observe phenomena before the clinical significance is known. For 
example, radiologists are increasingly encountering unidentified bright objects, or UBOs, in magnetic resonance images (MRI) of the brain. A study of MRI scans of healthy children found $13 \%$ with "abnormalities." However, on follow-up examination and after re-scanning 24 months later, none of these UBOs was found to be of clinical significance. ${ }^{4}$ In addition, the rates of incidental findings in neuroimaging vary widely, including reports of $20 \%$ of scans with incidental findings among asymptomatic individuals ${ }^{5}$ or even $40 \%$ among research participants. ${ }^{6}$ However, much lower rates of findings with clinical significance suggest the possibility of high false-positive rates. ${ }^{7}$ Results like these suggest that applying these new diagnostic tools in a clinical context prematurely may be unethical because it increases the potential for harm to patients, either because of over-treatment, under-treatment, unwarranted labeling and stigmatization, or a false sense of security.

Fourth, despite our current lack of a comprehensive understanding of the implications of microarray analysis, the large number of loci analyzed will create an unprecedented volume of information for patients, counselors, and health care providers. This makes it very challenging to envision what adequate informed consent means and how to achieve it.

It is important to reiterate that these issues are not new to medical geneticists or genetic counselors. However, they do raise issues for consideration by researchers who are using the technology, especially in the examination of complex traits, because of the implications of the findings for later clinical application and translation of the technology.

\section{ISSUES ARISING WHEN ARRAY CGH IS APPLIED TO RESEARCH ON COMPLEX PHENOTYPES}

There are several ethical issues specifically raised by the research application of array CGH to complex phenotypes. The broad ethical principles generally used to evaluate research on human subjects that are most relevant are autonomy, beneficence, and non maleficience. However, a recently articulated set of criteria proposed by Emanuel et al. ${ }^{8}$ for what makes clinical research ethical is perhaps a more useful framework because of its comprehensiveness and specificity in operationalizing basic principles in terms of the conduct of clinical research. These criteria include: value to health and knowledge, scientific validity, favorable risk:benefit ratio, informed consent, and respect for enrolled subjects. These requirements and the ethical principles that underlie them are relevant to evaluating the ethics of research studies on microarrays and complex traits.

However, we would add to this set of criteria by suggesting that the issues raised by this kind of research extend beyond the question of whether the design and conduct of the research itself is ethical, to the presentation and interpretation of the research results. This is especially relevant in the context of complex traits. Array CGH provides new opportunities to look for the genetic components of diseases and traits for which other techniques have been less successful. Complex traits, with multiple environmental and genetic risk factors, have not successfully yielded to linkage or candidate gene approaches, and only recently are genome-wide scans being applied in the attempt to characterize the genes involved. This failure to identify causal genes is particularly evident for traits that are involved in neurodevelopment and cognition, such as autism and mental retardation. Many of these traits do not have clear patterns of heritability in families; yet there is a strong desire on the part of parents and clinicians to understand the etiology, identify a clear diagnostic with clear predictive value, and avoid having children born with the condition.

In the context of this failure to convincingly identify genes, new ways of understanding genomic variation and new technological tools are exciting because of their potential promise for yielding answers where few have previously come. It is for this reason that many research groups are moving quickly to apply these new approaches to samples from complex traits.

However, in the midst of this flurry of activity, there are some important points to consider in research. It is important not to oversell the results, billing copy number variants (CNVs) as causal, without evidence and without explaining the limitations of the study. Overhyped, noncontextualized conclusions can lead to misperceptions in the genetics community and among patients and parents. Parents or pregnant couples, especially those who already have a child with or a family history of a complex disorder, may come into their physician's office or genetics clinic requesting array CGH testing, thinking that it will provide definitive answers about risk of complex traits. The research community can maximize benefit and minimize risk to patients and families, not just those in their studies but also those who may be affected by their research results, by developing and adhering to rigorous standards of evidence in the study of complex traits using array CGH and appropriate generalization from study results.

\section{ARRAY CGH/COPY NUMBER VARIATION RESEARCH IN AUTISM}

An examination of the research literature describing the application of array CGH in autism illustrates the use of this technology to examine a complex trait. Although array CGH is the technique used most often in clinical practice, the CNVs that it detects can also be identified by other techniques, such as various whole-genome genotyping methods. Therefore, throughout this article we refer to techniques used to study CNVs broadly, including but not limited to array CGH.

To make specific recommendations about the ethical implications of this kind of research, we analyzed publications in PubMed of original studies of autism using array CGH (using keywords "autism" and "array" or "copy number") published April 2005 through April 2007. This search yielded 14 publications that fell into three main categories.

The first category contained studies explicitly focusing on the association between copy number variants and autism, either idiopathic or syndromic. ${ }^{9-12}$ These studies ranged in population/sample size, from the largest at 6709 samples, ${ }^{10}$ an intermediate population with 389 samples, ${ }^{9}$ and two smaller populations of 29 samples $^{11}$ and six samples. ${ }^{12}$ 
A second category focused on genes or genomic regions either with some evidence of association with autism themselves or with syndromes known to have autistic phenotypes. ${ }^{13-19}$ These included regions in chromosome 15q, including those involved in Prader-Willi and Angelman syndromes, ${ }^{14-17}$ Williams-Beuren syndrome, ${ }^{19}$ patients with corpus callosum anomalies, ${ }^{18}$ and patients with autism itself. ${ }^{13}$

The third category included studies identifying novel deletions or deletion syndromes in single individuals or very small groups of individuals that included autistic phenotypes. ${ }^{20-22}$ These studies resulted from applications of array CGH in larger studies of autism, ${ }^{19}$ idiopathic intellectual disability, ${ }^{21}$ and genetics clinical practice. ${ }^{22}$ They included deletions on chromosomes 2, 21 17, 20 and the X chromosome. ${ }^{22}$

Our analysis of these articles suggest five points to consider regarding the application of the emerging technology of array CGH to research on complex traits like autism, which are aimed at a broad audience: clinicians and researchers reading and interpreting these or other published studies; researchers planning or writing the results of similar studies; peer reviewers reviewing articles or grant proposals; institutional review board members; clinicians or genetics counselors whose clients may request array CGH testing; and patients, families, and advocacy groups trying to understand how array CGH fits more broadly into research, diagnosis, and treatment. Although these points were generated from studies of autism, they could also apply to studies using array CGH and similar techniques to examine other complex traits.

\section{EVALUATION OF CRITERIA FOR AND LANGUAGE AROUND DETERMINING CAUSAL RELATIONSHIPS}

The studies we examined all attempted, to varying degrees, to make statements about causal relationships between copy number variations and autism. These statements were based on the criteria that the authors used for evaluating causation. A closer examination of these causal criteria reveals substantial heterogeneity and inconsistency. At least one article stated that all de novo alterations, or alterations identified in affected individuals but not in their parents or unaffected siblings, must be causal, based on the existing causal criteria used when detecting and interpreting large microscopic changes using karyotyping or FISH. ${ }^{9}$ It is not clear whether this standard is appropriate for smaller, potentially more common changes like those detected with array CGH. Other articles stated that the absence of a CNV in unaffected family members or in controls was evidence of causation. ${ }^{9-12}$ Still others compared the frequency of CNVs detected in cases compared with controls and cited a statistically significant difference in the number of de novo CNVs as evidence of causation. ${ }^{9}$

Without more data on the frequency of these kinds of changes, de novo or inherited in the population, it is difficult to make conclusive statements about causation. For sequence variations and microscopic changes, about which much more is known, variants can be more easily distinguished among those recognized or likely to be a cause of a disorder, those that are not likely to be causal and those of unknown clinical significance. ${ }^{23}$ However, at this stage of research, the finding of a CNV not previously identified does not in itself constitute evidence for pathogenicity.

The articles also vary in the language and terms they use to describe causal relationships and clinical relevance. Although the use of language may seem like an issue of mere semantics, it has powerful societal implications for diagnosis and identity for people who have autism, a disease for which no genes or other causes have been consistently identified. The recently published article by Sebat et al. in Science represents the extreme of confidence about causation, with the title: "Strong association of de novo copy number mutations and autism." The authors also state, "the strong association of de novo CNVs with ASD is consistent with such mutations being a primary cause in most cases rather than merely contributory." 9 These authors base their evidence of causation on the difference in CNVs identified in cases and controls, stating, "Since the difference in rate between autism and control is so marked, we can make a fair presumption that many of the lesions we observe are contributory to the disorder." However, this difference was only $10 \%(n=12)$ in cases compared with $2 \%{ }^{5}$ in controls, which was statistically significant using their analytic approach, but it is arguable whether "marked" or "significant" translates to a broader contribution to the disorder. ${ }^{9}$

A study by Jacquemont et al. published in $2006^{11}$ applied array CGH to 29 patients presenting with syndromic autism spectrum disorders (ASD), or ASD associated with other clinical features such as facial dysmorphism, limb or visceral malformations, and growth abnormalities. They referred to the identified CNVs as "clinically relevant rearrangements" and "pathogenic" because they were not previously identified in normal variants in the general population and were either de novo, or not present in either unaffected parent, or occurred only on a maternal $\mathrm{X}$ chromosome that was then inherited by an affected son. None of the variants were recurrent or overlapped in the eight patients.

Other articles are more cautious in the use of language around causation. They state that their goal is to identify potential candidate genes for autism, which would then need to be examined in different, larger, and more generalized populations. ${ }^{19}$ Others cite the need to exclude known rare causes of ASD to reduce the noise in linkage studies, candidate genes studies, and whole-genome studies and allow for the easier identification of candidate genes. ${ }^{10}$

The language used to describe causation is important because it affects the reception of the work by researchers, clinicians, the media, and patients and families. Perhaps the most important challenge in evaluating and describing causal relationships for complex diseases like autism relates to the frequency of variants and their impact on common diseases. Researchers ought to interpret their results in light of the possible population attributable risk (PAR), or the proportion of disease in the population explained by these changes. There is a meaningful difference between the suggestion that a gene may be involved in the biology of a common complex disease like 
autism, based on the discovery of a rare variant, and the conclusion that it plays an important or significant role in causing the disease more broadly. There is great danger that clinicians and families may be misled to believe that most or a large proportion of autism is caused by CNVs and is detectable using array CGH technologies, or that the detection of CNVs in children is tantamount to a genetic diagnosis. These misunderstandings can only be avoided by clear attention to language and conclusions by researchers and journals.

\section{EVALUATION OF STUDY DESIGN, INCLUDING THE SELECTION OF POPULATION AND SAMPLE SIZE}

It is important to examine the stated hypothesis of the authors, not only in the article of interest, but also in the original study. Studies using arrays or similarly new technologies are very often performed using samples collected in studies designed for different purposes. The challenge is that these analyses are limited by the original study design in ways that can affect their ability to accurately answer questions using the new technologies and approaches. The original study design and population size may limit statistical power to detect differences among cases and controls, multiplex or simplex families, or even phenotypic subgroups. Because we do not know with confidence the rate of CNVs in the general population, it is difficult to even estimate the sample size necessary to make these comparisons to determine causality.

The clinical and phenotypic definition of the cases is also variable among studies. In the case of autism, it is important to consider what diagnostic criteria were used for recruitment and whether the study is focused on idiopathic or syndromic autism. How representative are the cases of the majority of autism in the population? Are the cases included in the study from multiplex or simplex families? What kinds of conclusions are made about the phenotypic differences and causality? Additionally, what controls, if any, are examined? Are controls unaffected family members or unrelated individuals? For unaffected siblings, were they evaluated for the presence of any autism-related phenotypes? All of these questions affect the interpretability and generalizability of the results.

Early decisions in study design and data collection may also affect other aspects of array CGH analysis. Genomic DNA may not be available for any samples, particularly when DNA banks are being used as sources; therefore, researchers may not be able to confirm that the CNVs are not just an artifact of immortalization of cell lines. Cell lines from affected patients may have been treated differently than those of control samples, including more cell line passages, perhaps leading to an increase in chromosomal changes that are artifacts of immortalization. Furthermore, researchers may not have collected genomic DNA from parents or unaffected siblings; therefore, it may not be possible to determine whether CNVs are inherited or occur de novo.

These kinds of questions are not new. They are foundational principles of genetics and epidemiology and have long been applied to association studies. The editors of Nature Genetics summarized many of these points in a 1999 editorial on criteria for the publication of association studies. ${ }^{24}$ Although studies applying array CGH to complex diseases like autism may not use all the same principles as association studies, the level of rigor required for statements about causality should be similar.

\section{EVALUATION OF THE ARRAY TECHNOLOGIES USED}

There are several technologies and platforms used to evaluate copy number variations. These include ROMA, ${ }^{9} \mathrm{BAC}$ arrays, ${ }^{11,16-19,21}$ SNP arrays, ${ }^{10,12,14,20}$ and other whole-genome genotyping technologies. Each of these platforms uses a different approach to choosing what markers to measure, the experimental processes involved, the potential biases to include or exclude certain parts of the genome or kinds of sequence, and the density of the genome covered. Each has different measures of accuracy and reliability, even among laboratories, and each involves complex statistical analyses. Large studies estimating the range and frequency of copy number variation in normal populations also use some of these platforms preferentially, sometimes resulting in varying rates of detection, which affects the interpretation of findings in disease populations. All studies of CNVs should address each of these points and be clear that this variability among techniques makes replication among populations and platforms more critical in confirming causal relationships.

\section{CONSIDERATION OF STATEMENTS ABOUT GENERALIZABILITY AND LIMITATIONS}

Researchers publishing results using CNV and array CGH should remain cautious about the generalizability of their results and should follow the convention of linkage and association studies and call for replication of the results in other populations and using other platforms. Furthermore, they should not be misleading about the distance and ease of taking a single finding about $\mathrm{CNV}$ to greater biological understanding of causality or clinical application. Overstatement is of concern because of the possibility of encouraging the "therapeutic misconception" 25 while array CGH is in early stages of application in diagnostic uses.

For example, Sebat et al. wrote, "when an individual gene candidate can be identified, because the mutation affects a single gene or a small number of functional candidates, a straightforward path to validation can be planned, involving sequencing and higher resolution CNV analysis in additional samples." "We argue that this statement is overly optimistic. Such a path is only "straightforward" if other samples carry the same or overlapping rare CNV changes, or if changes in an affected gene or genes account for a large proportion of autism, which is unlikely given the findings of other genetic studies over the last decade. By contrast, the Autism Genome Project Consortium is more circumspect about the generalizability of their results, stating: "Without other information, this finding might not be especially meaningful, in particular because of the prevalence of CNVs in the genome." 10 We suggest that, particu- 
larly at this nascent stage of the application of these technologies to complex traits, a more specifically cautious approach and intentional statements about the need for replication is merited.

\section{CONSIDERATION OF ETHICAL IMPLICATIONS FOR STUDY SUBJECTS, INCLUDING CONSENT AND RETURN OF RESULTS TO PARTICIPANTS}

There are some additional specific ethical issues for study subjects involved in the research applications of array CGH. First, did participants give consent to participate in a study using array CGH? Most journals require that authors describe investigational review board approval for their research and state that participants signed consent forms. However, many genetics studies, not just those of array CGH, use samples that were collected much earlier. It is unlikely that the consent forms or processes described new genome-wide technologies like array CGH, or their ability to identify potentially pathogenic variations that might cause diseases beyond the original scope of research, and might have implications for reproductive decision-making. The risks and benefits of array CGH research on complex disease may be different than the risks and benefits of other genetic research.

Second, most array CGH findings require validation on genomic DNA samples, yet many studies do not have genomic DNA available and only have lymphoblastoid cell lines available for analysis. Investigators must choose either not to validate findings or to re-contact participants to obtain genomic DNA. Such recontacting is not ethical if the informed consent says that participants will not be re-contacted or if the investigators are using samples from a databank and are not the original investigators and therefore should not have access to identifiable information. Even if consent forms and investigator relationships made re-contact ethically acceptable, it would be challenging to re-contact participants without alarming them about the reason.

Third, it is important to consider whether the use of array CGH might ever require investigators to re-contact subjects under any circumstances because of the pathogenic importance of any finding, even an incidental finding. Such findings may include, for example, CNVs that are known to be associated with severe conditions for which some preventive therapy might be appropriate, or for which cautious reproductive decisions might be warranted (for example, large changes on the $\mathrm{X}$ chromosome in the mother). If CNVs known to be associated with clinically significant conditions are included in arrays used for research, it is important for researchers to consider beforehand whether and how to convey this information to subjects and how subjects should consider the possibility of receiving such information during the consent process. To the extent that array CGH is used to evaluate chromosomal integrity generally (as opposed to targeted analysis of specific loci), it may generate findings of known or likely clinical significance, such as a large deletion, even if they were not specifically sought as part of the research question, or even if the chromosomal abnormality is not known to be the cause of any partic- ular phenotype of research interest. In these cases, the research team should have plans for results to be validated and provided to the subject by a CLIA-certified lab. However, we realize that some of these findings may be ambiguous in terms of clinical validity and that a CLIA-certified lab that can perform an appropriate test to validate a research finding may not exist. In these cases, the nature of the finding is likely to be too preliminary to offer to research subjects.

All of these ethical concerns require careful consideration during study design and implementation. Because of their complexity, researchers might want to consider involving clinical geneticists, genetics counselors, or research ethics consultants ${ }^{26}$ in the study design and analysis processes. We would argue that these concerns also require careful consideration in the publication itself, and on the part of journal reviewers. Discussion of practical ways to address these concerns will also increase the ability of other researchers to address them.

\section{ACKNOWLEDGMENTS}

The authors were supported by Grant P50HG003389 from the National Human Genome Research Institute.

\section{References}

1. Secretary's Advisory Committee on Genetic Testing. Enhancing the oversight of genetic tests: recommendations of the SACGT. Bethesda, MD: National Institutes of Health, 2000. Available at: http://www4.ed.nih.gov/oba/sacgt.htm. Accessed May 7, 2007.

2. Check E. Screen test. Nature 2005;438:733-734.

3. Grody W. Ethical ramifications of genetic analysis using DNA arrays. In: Rampal JB, editor. DNA arrays: Methods and protocols. Totowa, NJ: Humana Press, 2001: 53-69.

4. Kumra S, Ashtari M, Anderson B, Cervellione KL, et al. Ethical and practical considerations in the management of incidental findings in pediatric MRI studies. J Am Acad Child Asolesc Psychiatry 2006;45:1000-1006.

5. Katzman GL, Dagher AP, Patronas NJ. Incidental findings on brain magnetic resonance imaging from 1000 asymptomatic volunteers. JAMA 1999;282:36-39.

6. Illes J, Kirschen MP, Karetsky K, Kelly M, et al. Discovery and disclosure of incidental findings in neuroimaging research. J Magn Reson Imaging 2004;20:743-747.

7. Illes J, Kirschen MP, Edwards E, Stanford LR, et al. Ethics: incidental findings in brain imaging research. Science 2006;311:783-784.

8. Emanuel EJ, Wendler D, Grady C. What makes clinical research ethical? JAMA 2000;283:2701-2711.

9. Sebat J, Lakshmi B, Malhotra D, Troge J, et al. Strong association of de novo copy number mutations with autism. Science 2007;316:445-449.

10. The Autism Genome Project Consortium. Mapping autism risk loci using genetic linkage and chromosomal rearrangements. Nat Genet 2007;39:319-328.

11. Jacquemont M-L, Sanlaville D, Redon R, Raoul O, et al. Array-based comparative genomic hybridization identifies high frequency of cryptic chromosomal rearrangements in patients with syndromic autism spectrum disorders. J Med Genet 2006;43: 843-849.

12. Baron CA, Liu SY, Hicks C, Gregg JP. Utilization of lymphoblastoid cell lines as a system for the molecular modeling of autism. J Autism Dev Disord 2006;26: 973-982.

13. Durand CM, Betancur C, Boeckers TM, Bockmann J, et al. Mutations in the gene encoding the synaptic scaffolding protein SHANK3 are associated with autism spectrum disorders. Nat Genet 2007;39:25-27.

14. Baron CA, Tepper CG, Liu SY, Davis RR, et al. Genomic and functional profiling of duplicated chromosome 15 cell lines reveal regulatory alterations in UBE3A-associated ubiquitin-proteasome pathway processes. Hum Mol Genet 2006;15:853-869.

15. Koochek M, Harvard C, Hildebrand MJ, Van Allen M, et al. 15q duplication associated with autism in a multiplex family with a familial cryptic translocation $\mathrm{t}(14$; 15)(q11.2;q13.3) detected using array-CGH. Clin Genet 2006;69:124-134.

16. Sahoo T, Shaw CA, Young AS, Whitehouse NL, et al. Array-based comparative genomic hybridization analysis of recurrent chromosome $15 \mathrm{q}$ rearrangements. $A m$ J Med Genet 2005;139A:106-113. 
17. Sahoo T, Peters SU, Madduri NS, Glaze DG, et al. Microarray based comparative genomic hybridization testing in deletion bearing patients with Angelman syndrome: genotype-phenotype correlations. J Med Genet 2006;43:512-516.

18. Sherr EH, Owen R, Albertson DG, Pinkel D. Genomic microarray analysis identified candidate gene loci in patients with corpus callosum anomalies. Neurology 2005;65: 1496-1498.

19. Edelmann L, Prosnitz A, Pardo S, Bhatt J, et al. An atypical deletion of the WilliamsBeuren syndrome interval implicates genes associated with defective visuospatial processing and autism. J Med Genet 2007;44:136-143.

20. Nakamine A, Ouchanov L, Jiménez P, Manghi ER, et al. Duplication of 17(p11.2p11.2) in a male child with autism and severe language delay. Am J Med Genet Part A 2007. In Press.
21. Rajcan-Separovic E, Harvard C, Liu X, McGillvray B, et al. Clinical and molecular cytogenic characterization of a newly recognized microdeletion syndrome involving 2p15-16.1. J Med Genet 2007;44:269-276.

22. Bonnet C, Grégoire MJ, Brochet K, Raffo E, et al. Pure de-novo $5 \mathrm{MB}$ duplication at Xp11.22-p11.23 in a male: phenotype and molecular characterization. J Hum Genet 2006;51:815-821.

23. ACMG Laboratory Practice Committee Working Group. ACMG recommendations for standards for interpretation of sequence variations. Genet Med 2000, 2:302-303.

24. Anonymous. Freely associating. Nat Genet 1999;22:1-2.

25. Appelbaum P, Roth L, Lidz C. The therapeutic misconception: informed consent in psychiatric research. Int J Law Psychiatry 1982:5:319-329.

26. Pilcher H. Dial 'E' for ethics. Nature 2006;440:1104-1105. 